\title{
High-sensitivity temperature sensor based on microstructured fiber outside coated with gold and PDMS films
}

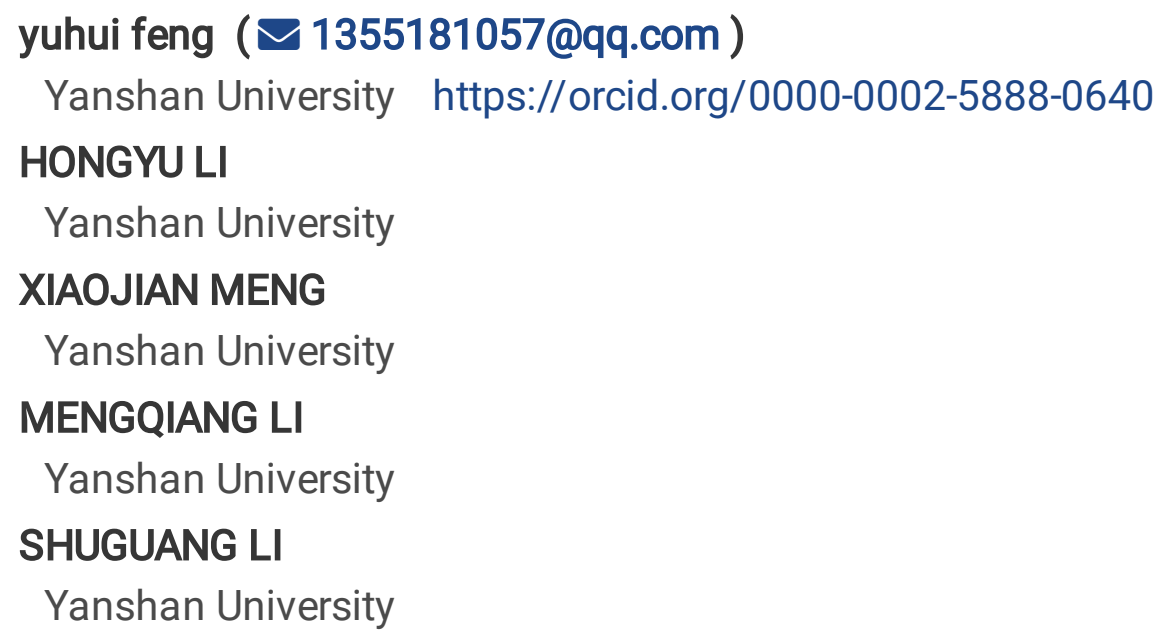

\section{Research Article}

Keywords: Microstructured Fiber, Surface Plasmon Resonance, Polydimethylsiloxane (PDMS), Temperature Sensor, High-sensitivity

Posted Date: February 14th, 2022

DOI: https://doi.org/10.21203/rs.3.rs-1293884/v1

License: (c) (1) This work is licensed under a Creative Commons Attribution 4.0 International License. Read Full License 


\title{
High-sensitivity temperature sensor based on microstructured fiber outside coated with gold and PDMS films
}

\author{
YUHUI FENG, HONGYU LI, XIAOJIAN MENG, MENGQIANG LI, \\ SHUGUANG LI*
}

State Key Laboratory of Metastable Materials Science \& Technology and Key Laboratory for Microstructural Material Physics of HebeiProvince, School of Science, Yanshan University, Qinhuangdao, 066004, PR China

*Corresponding author: shuguangli@ysu.edu.cn

\begin{abstract}
s
A high-sensitivity temperature sensor based on microstructured fiber (MSF) outside coated with gold and Polydimethylsiloxane (PDMS) films has been presented in this paper. The gold film is coated outside the fiber cladding, wich is used to excite the surface plasmon resonance (SPR), and PDMS is a kind of substance with high thermo-optical coefficient and easy to be cured, the temperature response has been effectively improved by depositing PDMS film outside the gold film. The influences of the parameters on the sensing characteristics are investigated by using the finite element method (FEM). Simulation results show that the average temperature sensitivity is up to $9.287 \mathrm{~nm} /{ }^{\circ} \mathrm{C}$ in the range of $-20^{\circ} \mathrm{C}-40^{\circ} \mathrm{C}$. Moreover, compared with other designs, the fully coated structure on the outside of MSF enables temperature-sensitive medium to be in direct contact with the environment, which reduces the difficulty of internal coating and filling in the air-holes of MSF. The optimized process of structure in this study provides an effective method, and the temperature sensor based on MSF has good application prospects in the fields such as medical treatment, biochemical detection, and intelligent monitoring.
\end{abstract}

Keywords : Microstructured Fiber; Surface Plasmon Resonance; Polydimethylsiloxane (PDMS); Temperature Sensor; High-sensitivity 


\section{Introduction}

Photonic crystal fiber (PCF), also known as microstructured fibers (MSF) that have air holes extending along the fiber in its cross section [1-3]. PCF have been extensively applied to sensors [4], filters [5], lasers [6], and other optical devices due to the flexible structural design and many distinctive characteristics [2]. Since the distinguished performance of surface plasmon resonance (SPR) technology in the sensing domain, SPR technology has been combined with PCF to increase the function of the fiber [7]. Compared to conventional fiber sensors, SPR-PCF sensors are obviously optimized in terms of cross-sensitivity and coupling loss. In addition, it has qualities of wide operating wavelength range, high sensitivity, and multi-parameter measurement [8-10]. Owing to surface plasmon polaritons are sensitive to the external environment and easily changing transmission constants of sensor, SPR-PCF sensors have aroused tremendous interest in temperature measurement, medical diagnostics and more [10].

Temperature measurement plays an indispensable role in industrial production, environmental monitoring and biological fluids. At present, electrical temperature sensor has been developed and matured. But as technology advances, fiber temperature sensor based on combination of fiber and SPR has attracted people's attention. Fiber temperature sensors have gradually substituted electrical temperature sensors because of its preponderance of long-distance transmission and design flexibility. In 2014, Yang et al. [11] proposed and experimentally demonstrated a PCF temperature sensor based on SPR, which achieves temperature sensing by filling PCF with silver nanowires and liquid. Experimental results show that the sensitivity was $-2.08 \mathrm{~nm} /{ }^{\circ} \mathrm{C}$, whereas the operation procedure for filling in PCF was cumbersome. In 2015, Zhao et al. [12] presented a PCF-SPR sensor for temperature detection by using a temperature-sensitive liquid as an intermediate and combined with fiber SPR structure. The sensing element is fabricated by packaging a silver-coated fiber probe into a capillary filled with thermosensitive anhydrous ethanol. This proposed sensor achieves the sensitivity of $1.5745 \mathrm{~nm} /{ }^{\circ} \mathrm{C}$ between $35-70^{\circ} \mathrm{C}$. In 2016 , Luan et al. [13] presented a SPR sensor, which can simultaneously realize refractive index (RI) and temperature sensing in an exposed-core MSF. Two orthogonal passageways coated with silver layers are designed in cladding air holes, one of which is filled with thermosensitive liquid to distinguish the variation of analyte RI and temperature. The corresponding sensitivity was $6.18 \mathrm{~nm} /{ }^{\circ} \mathrm{C}$ as the temperature changed from 26 to $43^{\circ} \mathrm{C}$. The same year, Lu et al. [14] accomplished sensing by filling liquid crystals into a hollow-core fiber which was coated with a gold film on the inner wall. The sensitivity was $4.72 \mathrm{~nm} /{ }^{\circ} \mathrm{C}$ in the range of 20 to $34.5^{\circ} \mathrm{C}$. Subsequently, Zhu et al. [15] proposed a novel PCF sensor by combining SPR principle with defect coupling mechanism. Two air holes are determined in cladding, metal films are deposited on one of inner surfaces while the diameter of the other air hole is changed. Magnetic fluid material is filled in these two air holes. By analyzing the relationship between RI of magnetic fluid with its temperature and magnetic field, simultaneous sensing of temperature and magnetic field are realized. Liu [16] presented a new core-cladding symmetrical structure PCF in 2019, which fills all air holes of a group of core-cladding with ethanol. Temperature measurement is achieved due to RI of ethanol changes with temperature. Simulation results show that the sensitivity of $1 \mathrm{~cm}$ fiber meets $3.21 \mathrm{~nm} /{ }^{\circ} \mathrm{C}$ in the area from 20 to $25^{\circ} \mathrm{C}$. According to previous studies, most existing fiber temperature sensors selected to be filled with temperature-sensitive materials in PCF. Since infiltrating liquid into air holes or coating metal on the inner wall is comparatively inconvenient in practical approach, fabrication of sensors described above is rather complicated. In addition, filling media are mainly alcohol, benzene or liquid crystal. These materials respond significantly to changes in temperature but poisonous or volatility, which is not conducive to preservation and recycle of sensors. In order to surmount the above problems, this study chooses to wrap the double-layer membrane immediately outside $\mathrm{PCF}$, which reduces complexity of the structure and is more sensitive to external surroundings.

This paper numerically reports a high-sensitivity temperature sensor by using commercial software COMSOL Multiphysics, finite element method (FEM), with anisotropic perfectly matched layers (PML). First of 
all, Gold with high SPR effect is deposited outside MSF cladding with chemical vapor deposition (CVD), owing to its chemical stability and is less likely to be oxidized in presence of temperature sensing medium. Then, PDMS is wrapped outside the gold film through the curing process, which makes it in direct contact with the environment to be measured and reduce the manufacturing difficulty. By optimizing the parameters, results show that the identification ability of sensor for temperature is improved effectively. It's found that the average sensitivity of sensor is $9.287 \mathrm{~nm} /{ }^{\circ} \mathrm{C}$, and the maximum can reach $13 \mathrm{~nm} /{ }^{\circ} \mathrm{C}$. Finally, the influences of $\mathrm{MSF}$ parameters on resonance wavelength, loss value and sensitivity are analyzed. Owing to the simple structure and stable performance, the proposed MSF sensor has a chance of actually being manufactured with current technology.

\section{Structural design and theoretical analysis}

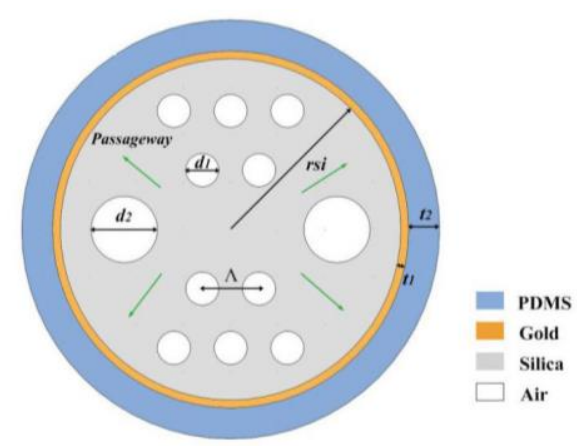

(a)

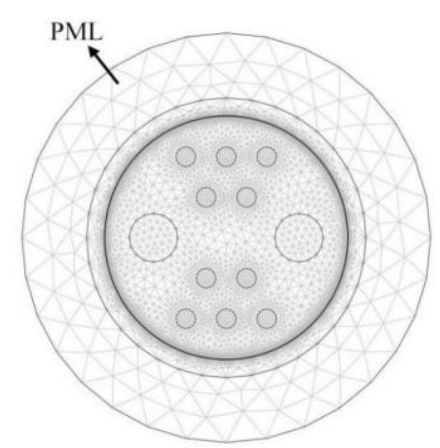

(b)

Fig.1. (a) Cross-section of the proposed MSF; (b) the meshing of computational region for the optimized structure: $\mathrm{d}_{1}=1.2 \mu \mathrm{m}, \mathrm{d}_{2}=3 \mu \mathrm{m}, \Lambda=2.5 \mu \mathrm{m}, \mathrm{t}_{1}=35 \mathrm{~nm}$ and $\mathrm{t}_{2}=1.1 \mu \mathrm{m}$.

The two-dimensional cross-sectional of the proposed MSF is depicted in Fig. 1(a). It consists of twelve air holes arranged in a hexagonal grid. In order to promote the surface plasmons propagate effectively on metal dielectric surface, air holes with two different diameters are designed. The large air holes are placed on both sides parallel to the fiber core while the small air holes are arranged on the upper and lower sides. Consequently, evanescent wave is transferred to gold film through passageways as shown by arrows in Fig. 1(a), which passageways radius is $\mathrm{r}=7.5 \mu \mathrm{m}$. The diameters of air holes are represented by $\mathrm{d}_{1}$ and $\mathrm{d}_{2}$, where $\mathrm{d}_{1}=1.2 \mu \mathrm{m}$, $\mathrm{d}_{2}=3 \mu \mathrm{m} ; \Lambda$ represents the horizontal distance between adjacent small air holes, which was determined to be $2.5 \mu \mathrm{m}$; A gold layer with diameter of $\mathrm{t}_{1}=35 \mathrm{~nm}$ is used as plasmonic material, which is deposited outside the MSF cladding by CVD method; Symbol $t_{2}$ expresses the thickness of sensing medium PDMS, which is wrapped on the gold surface and it is considered to be $t_{2}=1.1 \mu \mathrm{m}$; The proposed MSF is practically realizable through stack-and-draw fabrication means because of its simple structure. By commercial software COMSOL Multiphysics to investigate sensing properties of the proposed MSF. Fig. 1(b) indicates the meshing of the calculation region for optimized structure. To guarantee the computational accuracy, PML and scattering boundary condition are increased.

Pure silica is the background material of MSF whose dispersion coefficient is calculated by the Sellmeier equation [17] :

$$
n^{2}(\lambda)=1+\frac{B_{1} \lambda^{2}}{\lambda^{2}-C_{1}}+\frac{B_{2} \lambda^{2}}{\lambda^{2}-C_{2}}+\frac{B_{3} \lambda^{2}}{\lambda^{2}-C_{3}}
$$

Here, $n$ is the refractive index of fused silica, which is decided by incident light wavelength $\lambda$ in vacuum. $B_{1}, C_{1}$, $\mathrm{B}_{2}, \mathrm{C}_{2}, \mathrm{~B}_{3}, \mathrm{C}_{3}$ are known as Sellmeier constants.

The dielectric constant of gold is described by the Drude-Lorentz formula [18] :

$$
\varepsilon_{\mathrm{m}}=\varepsilon_{\infty}-\frac{\omega_{D}^{2}}{\omega\left(\omega+i \gamma_{\mathrm{D}}\right)}-\frac{\Delta \varepsilon \cdot \Omega_{L}^{2}}{\left(\omega^{2}-\Omega_{L}^{2}\right)+i \Gamma_{L} \omega}
$$

where $\varepsilon_{\mathrm{m}}$ expresses the permittivity of gold, and $\varepsilon_{\infty}=5.9673$ is the permittivity at high frequency. $\omega=2 \pi \mathrm{c} / \lambda$ indicates angular frequency of guided light where $\mathrm{c}$ is the velocity of light, $\omega_{\mathrm{D}}=2 \pi \times 2113.6 \mathrm{THz}$ refers to plasmon frequency, and $\gamma_{D}=2 \pi \times 15.92 \mathrm{THz}$ represents the damping frequency. $\Delta \varepsilon=1.09$ can be interpreted as a weighting factor, $\Omega_{\mathrm{L}}=2 \pi \times 650.07 \mathrm{THz}$ and $\Gamma_{\mathrm{L}}=2 \pi \times 104.86 \mathrm{THz}$ are 
frequency and spectral width of the Lorentz oscillator, respectively.

PDMS is silicone material with unique optical properties extensively applied to optical field, which has excellent qualities of wide operating range, non-toxic and chemically stable [19]. It has high thermo-optic coefficients and is sensitive to temperature change. At the same time, the extraordinary optical characteristics of PDMS are not affected after curing. Its effective refractive index varies with temperature as determined by the equation [20-22] :

$$
n_{P D M S}=n_{0}-\gamma \Delta \mathrm{T}
$$

In this expression, $n_{0}=1.421332$ is the effective refractive index of PDMS at $20^{\circ} \mathrm{C}, \gamma=-4.66 \times 10^{-4} /{ }^{\circ} \mathrm{C}$ is thermo-optical coefficient, $\Delta \mathrm{T}$ refers to temperature variation.

Confinement loss is an indispensable parameter while investigating MSF, which can be defined by the following equation [23] :

$$
\alpha=8.868 \times \frac{2 \pi}{\lambda} \operatorname{Im}\left(n_{\text {eff }}\right) \times 10^{-4}
$$

where the units of $\alpha$ are $\mathrm{dB} / \mathrm{cm}$. $\lambda$ is wavelength in vacuum and $\operatorname{Im}\left(n_{\text {eff }}\right)$ represents virtual part of the effective refractive index.

The capability of sensor can also be reflected by sensitivity, which can be calculated by wavelength interrogation method as follows [24] :

$$
S(\lambda)=\frac{\Delta \lambda_{\text {peak }}}{\Delta T}\left(\mathrm{~nm} /{ }^{\circ} \mathrm{C}\right)
$$

Here, $\Delta \lambda_{\text {peak }}$ represents the distance of resonance peak shift and $\Delta \mathrm{T}$ indicates the amount of temperature change.

As shown in Fig. 2, experimental setup for realize temperature sensing mainly includes the following three parts: broadband light source (BBS), temperature control chamber and optical spectral analyzer (OSA). BBS is used to transmit light into single mode fiber (SMF) and couple with MSF-SPR sensor. Then the sensor responds to temperature by changing the temperature of surroundings with temperature control chamber. Finally, transmission spectrum is tracked and displayed by OSA. Temperature changes will lead to variations in RIs of PDMS. Since different RIs will change the phase matching condition of fundamental mode and SPR mode result in loss peak shift. Therefore, temperature measurement is achieved by analyzing the relationship between temperature variation and resonance wavelengths drift.

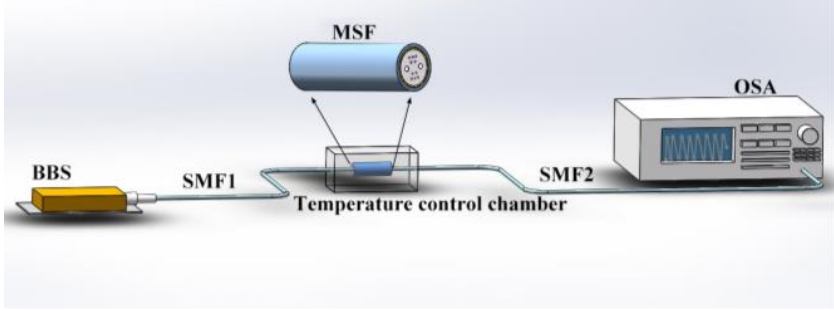

Fig.2. Schematic diagram of the experimental setup for the proposed MSF temperature sensor.

\section{Results and discussions}

A. Influences of different parameters on sensing characteristics

In this segment, the influences of structural parameters $d_{1}, \Lambda, t_{1}$, and $t_{2}$ on loss values, sensitivity and resonance wavelengths are discussed.
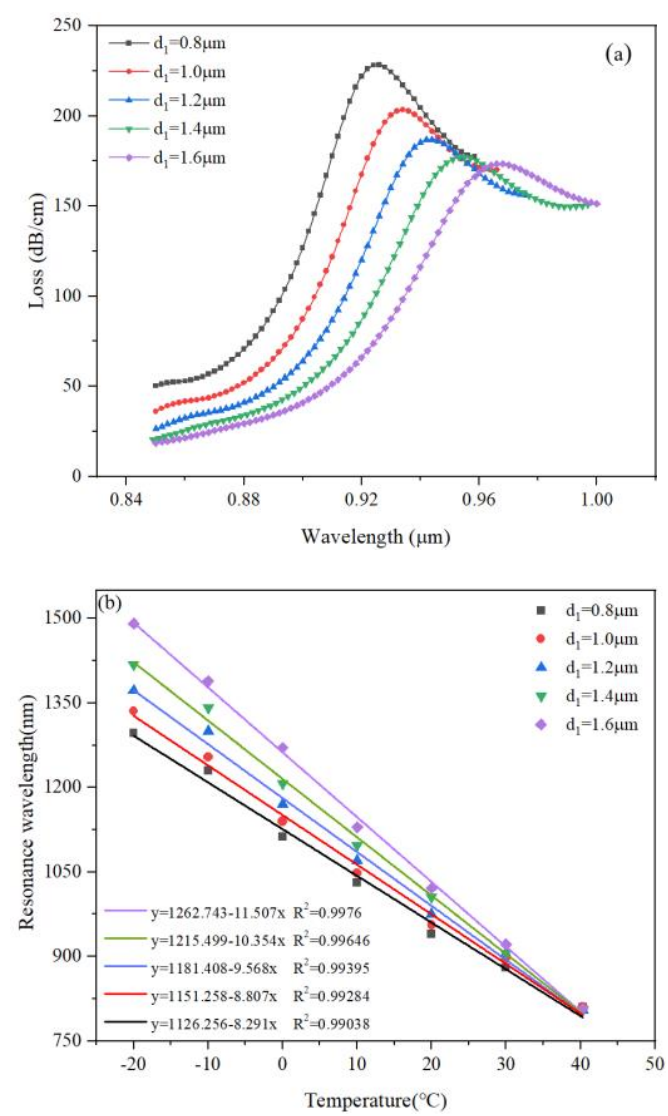

Fig.3. (a)Variation of loss spectrum at $20^{\circ} \mathrm{C}$ with different $\mathrm{d}_{1 \text {; }}$ (b)Variation of the resonance wavelengths with different $d_{1}$ in the range $-20 \sim 40^{\circ} \mathrm{C}$. 
Fig. 3(a) shows that the confinement loss relies on the operable wavelength by changing diameter of $\mathrm{d}_{1}$. It can be seen that with increases of $d_{1}$, the confinement loss decreases and resonance wavelength red-shifts, which is owing to the enhanced limitation of light. The energy transferred to gold surface decreases due to the enhancement of the bound light ability, which weakens the SPR phenomenon and reduces the loss value. Variations of the resonance wavelengths with different $d_{1}$ over the temperature ranges from $-20^{\circ} \mathrm{C}$ to $40^{\circ} \mathrm{C}$ as depicted in Fig. 3(b). It can be seen from Fig. 3(b) that when $d_{1}$ is fixed, resonance wavelength blue-shifts with increase of temperature. Average sensitivity is obtained about $\quad 8.291 \mathrm{~nm} /{ }^{\circ} \mathrm{C}, \quad 8.807 \mathrm{~nm} /{ }^{\circ} \mathrm{C}, \quad 9.568 \mathrm{~nm} /{ }^{\circ} \mathrm{C}$, $10.354 \mathrm{~nm} /{ }^{\circ} \mathrm{C}, 11.507 \mathrm{~nm} /{ }^{\circ} \mathrm{C}$, respectively, with the $\mathrm{d}_{1}$ increased from $0.8 \mu \mathrm{m}$ to $1.6 \mu \mathrm{m}$. Increasing $\mathrm{d}_{1}$ is instrumental in improving sensitivity, while half-peak width tends to wider and the shape of spectrum is too smooth. Therefore, $\mathrm{d}_{1}=1.2 \mu \mathrm{m}$ is the optimal value by considering the confinement loss and wavelength sensitivity.
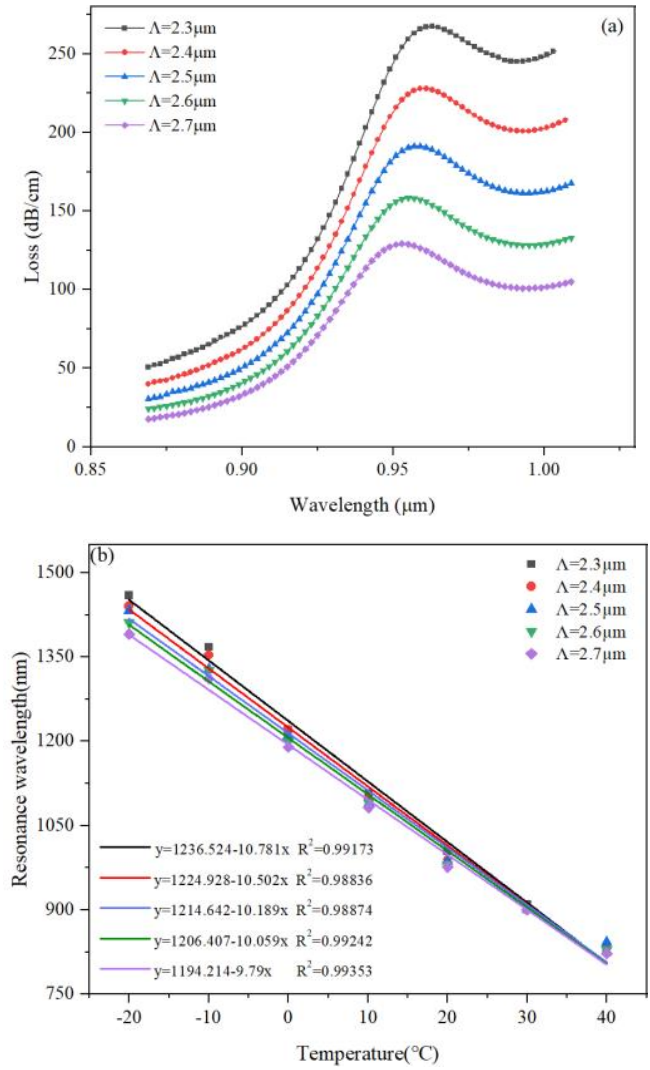

Fig.4. (a)Variation of loss spectrum at $20^{\circ} \mathrm{C}$ with different $\Lambda$; (b)Variation of the resonance wavelengths with different $\Lambda$ in the range $-20 \sim 40^{\circ} \mathrm{C}$.
Fig. 4(a) describes the variation of confinement loss spectrum for different $\Lambda$ at $20^{\circ} \mathrm{C}$. From Fig. 4(a), it can be concluded that the resonance wavelength blue-shifts when $\Lambda$ changes from $2.3 \mu \mathrm{m}$ to $2.4 \mu \mathrm{m}$ while peak loss decreases. The large $\Lambda$ narrows the transmission route of energy to the metal layer, which results in a weakened coupling between fundamental mode and SPP mode.

Variations of the resonance wavelengths with different $\Lambda$ over the sensing range are shown in Fig. 4(b). The maximum wavelength sensitivity of $10.781 \mathrm{~nm} /{ }^{\circ} \mathrm{C}$ is obtained at $\Lambda=2.3 \mu \mathrm{m}$, while the corresponding confinement loss is not conducive to practical application. All in all, by considering the peak loss and sensitivity $\Lambda$ is determined to be $2.5 \mu \mathrm{m}$.
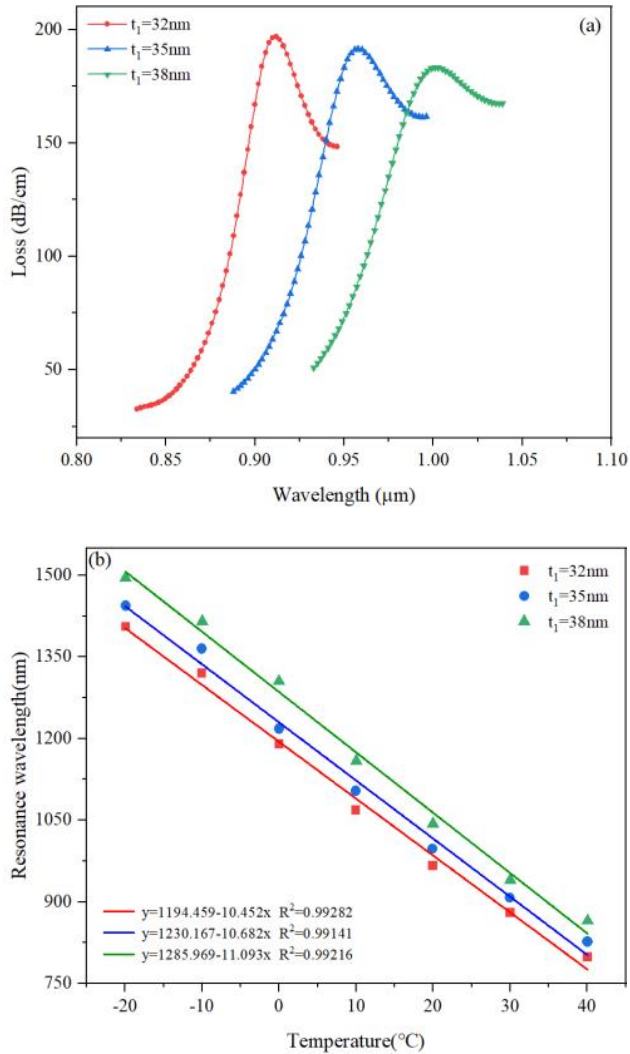

Fig.5. (a)Variation of loss spectrum at $20^{\circ} \mathrm{C}$ with different $t_{1}$; (b)Variation of the resonance wavelengths with different $\mathrm{t}_{1}$ in the range $-20 \sim 40^{\circ} \mathrm{C}$.

As observed in Fig. 5(a), the confinement loss depends on operable wavelength by changing $t_{1}$. The confinement loss decreases and the resonance wavelength red-shifts as $t_{1}$ increases. Since the effective refractive 
index of fiber core remains invariant, while the effective refractive index of SPP mode varies with $t_{1}$, which results in the shift of coupling position between two modes. The thickness of metal film will influence the decay rate of evanescent wave and spp mode. Thicker gold layer is responsible for loss value decreases and half-peak width increases. With the increase of gold layer, the energy of evanescent field decreases and confinement loss increases. The thickness of the gold layer also affects sensitivity of sensor, as depicted in Fig. 5(b). As seen in Fig. 5(b), sensitivity increases from 10.452 to $11.093 \mathrm{~nm} /{ }^{\circ} \mathrm{C}$ with the gold layer increases from $32 \mathrm{~nm}$ to $38 \mathrm{~nm}$. Taking into account sensitivity and confinement loss, $t_{1}=35 \mathrm{~nm}$ is determined as the optimum gold layer thickness of the sensor.
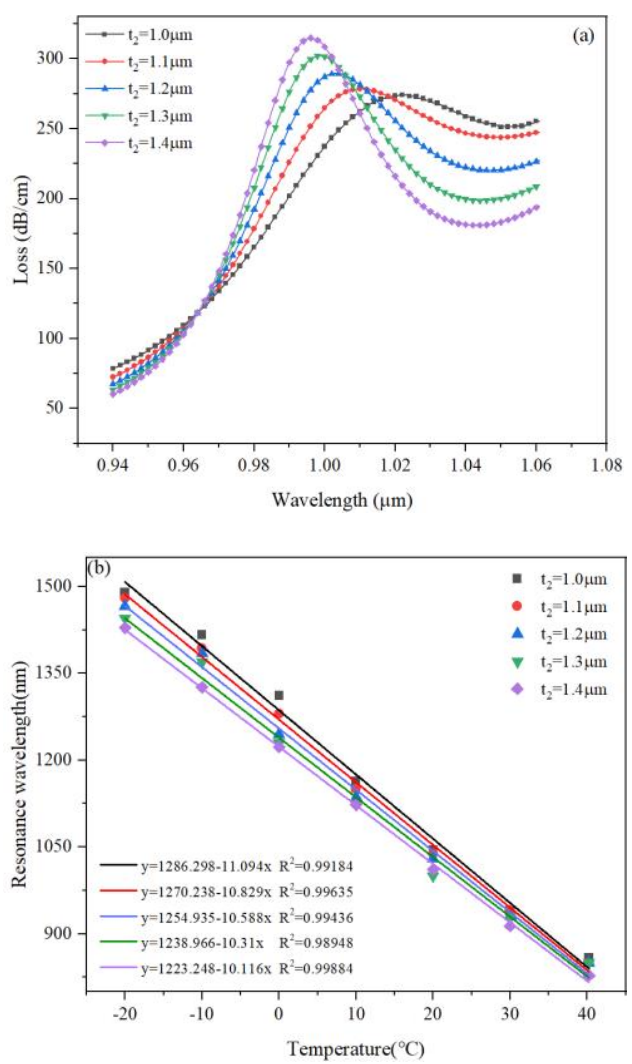

Fig.6. (a)Variation of loss spectrum at $20^{\circ} \mathrm{C}$ with different $\Lambda$; (b)Variation of the resonance wavelengths with different $\Lambda$ in the range $-20 \sim 40^{\circ} \mathrm{C}$.

The effect of varying sensor parameter $t_{2}$ on loss spectrum and sensitivity is illustrated in Fig. 6. Fig. 6(a) indicates that confinement loss increases significantly and blue-shifts as $t_{2}$ increases. In addition, the half-peak width greatly decreases and the spectral appears sharper. That is because the RIs of PDMS decreases with increase of temperature, which leads to the variation of phase matching wavelength. According to Fig. 6(b), sensitivities decreased from 11.094 to $10.116 \mathrm{~nm} /{ }^{\circ} \mathrm{C}$, when the $t_{2}$ increased from $1.0 \mu \mathrm{m}$ to $1.4 \mu \mathrm{m}$. In order to improve the sensing performance and meet practical applications, $\mathrm{t}_{2}=1.1 \mu \mathrm{m}$ was chosen as the optimum PDMS layer thickness. Finally the influence of each parameter on sensor performance is summarized, which is illustrated in Table 1.

Table. 1 The effects of different parameters on spectra and sensitivity.

\begin{tabular}{ccccc}
\hline $\begin{array}{c}\text { Parameter } \\
\mathrm{s}\end{array}$ & $\begin{array}{c}\text { Variation } \\
\text { range }(\mu \mathrm{m})\end{array}$ & $\begin{array}{c}\text { Loss } \\
\text { value }\end{array}$ & $\begin{array}{c}\text { Spectrum } \\
\text { shift }\end{array}$ & $\begin{array}{c}\text { Sensitivit } \\
\mathrm{d}_{1}\end{array}$ \\
$\Lambda$ & $0.8 \sim 1.6$ & Decrease & Red-shift & Increase \\
$\mathrm{t}_{1}$ & $0.032 \sim 0.038$ & Decrease & Red-shift & Increase \\
$\mathrm{t}_{2}$ & $1.0 \sim 1.4$ & Increase & Blue-shift & Decrease \\
\hline & & Decrease & Blue-shift & Decrease \\
B. Sensing & Performances & of & the & Proposed \\
Temperature Sensor & & &
\end{tabular}

From the above discussion, optimal parameters for this sensor were determined, which are observed in Table 2. Fig. 7(a) shows the confinement loss dependence on operable wavelength as temperature varies from $-20^{\circ} \mathrm{C}$ to $40^{\circ} \mathrm{C}$ and the temperature interval is $10^{\circ} \mathrm{C}$. At the resonance wavelength of 1380, 1280, 1150, 1030, 950, 890 , and $840 \mathrm{~nm}$, the corresponding losses are 405.45, 328.57, 267.46, 229.97, 201.7, 175.35, and $152.77 \mathrm{~dB} / \mathrm{cm}$. The changes of resonance wavelength at different temperatures and fit curves are depicted in Fig. 7(b). The equation of the linear fitting is determined as $\mathrm{y}=1168.28-9.287 \mathrm{x}$, where $\mathrm{y}$ and $\mathrm{x}$ represent the resonance wavelength and temperature, respectively. The slope $\mathrm{K}$ represents the average sensitivity of the sensor from $-20^{\circ} \mathrm{C}$ to $40^{\circ} \mathrm{C}$, which is $9.287 \mathrm{~nm} /{ }^{\circ} \mathrm{C}$. Linearity $\mathrm{R}^{2}$ is equal to 0.99114 . The maximum sensitivity of the sensor reaches $13 \mathrm{~nm} /{ }^{\circ} \mathrm{C}$ in the range of $-10^{\circ} \mathrm{C} \sim 0^{\circ} \mathrm{C}$. 

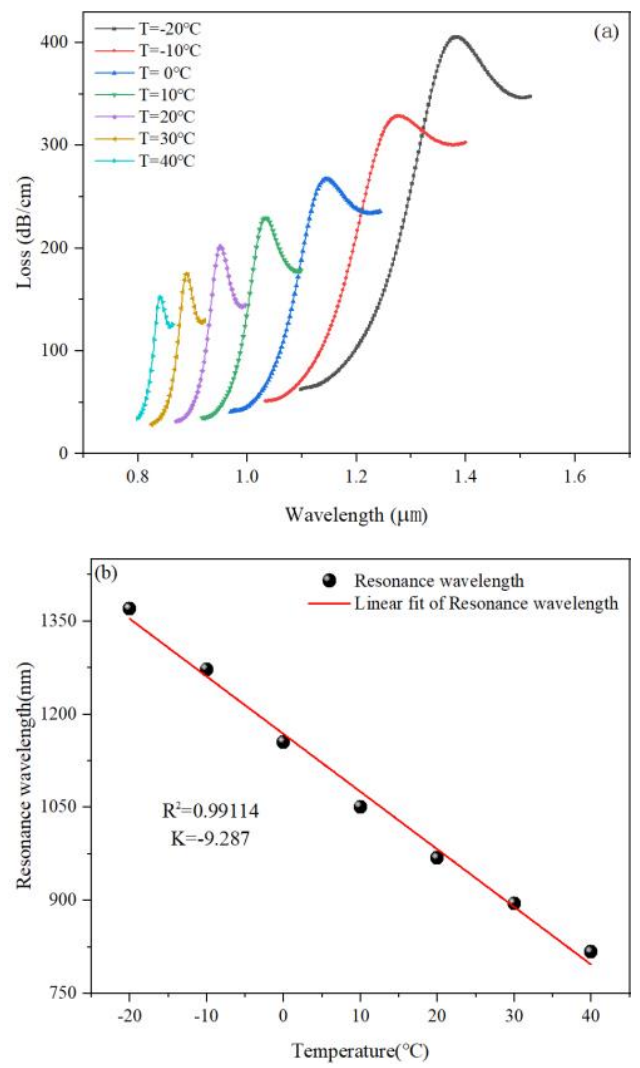

Fig.7. (a) Variation of loss spectrum; (b) Resonance wavelengths and its fit curve.

Table. 2 The value of the optimized parameters.

\begin{tabular}{ccccc}
\hline Parameters & $\mathrm{d}_{1}$ & $\Lambda$ & $\mathrm{t}_{1}$ & $\mathrm{t}_{2}$ \\
\hline value $(\mu \mathrm{m})$ & 1.2 & 2.5 & 0.035 & 1.1 \\
\hline
\end{tabular}

In addition to the criterion of sensitivity, measurement accuracy can also be regarded as intuitive reflection of sensor performance. The measurement accuracy determines the minimum temperature change sensed by sensor, which can be defined by the following expression [25] :

$$
R=\frac{\Delta T \Delta \lambda_{\min }}{\Delta \lambda_{\text {peak }}}\left({ }^{\circ} \mathrm{C}\right)
$$

where $\Delta \mathrm{T}$ refers to the temperature variation. The minimum detection accuracy of the spectrometer is $\Delta \lambda_{\min }$, which is generally taken as $0.1 \mathrm{~nm}$. $\Delta \lambda_{\text {peak }}$ expresses the wavelength shift. The proposed sensor is capable of detecting extremely small temperature changes with an accuracy of $0.01{ }^{\circ} \mathrm{C}$. A detailed comparison of the performance of our work with previous studies based on sensing range and wavelength sensitivity, which is illustrated in Table 3. These outcomes strongly highlighted the proposed sensor properties in the field of fiber sensing.

Table 3. Comparison of the proposed temperature sensor with previous studies.

\begin{tabular}{lllll}
\hline Reference & Sensing Type & Detection range $\left({ }^{\circ} \mathrm{C}\right)$ & $\Delta \mathrm{T}\left({ }^{\circ} \mathrm{C}\right)$ & Sensitivity $\left(\mathrm{nm} /{ }^{\circ} \mathrm{C}\right)$ \\
\hline$[26]$ & SPR & $0 \sim 50$ & 50 & 0.72 \\
{$[27]$} & Sagnac & $25 \sim 33$ & 8 & 1.65 \\
{$[13]$} & SPR & $26 \sim 43$ & 17 & 6.18 \\
{$[28]$} & Mach-Zehnder & $23.2 \sim 58.2$ & 35 & 1.83 \\
{$[29]$} & WGM & $25 \sim 45$ & 20 & 0.377 \\
This work & SPR & $-20 \sim 40$ & 60 & 9.287 \\
\hline
\end{tabular}

\section{Conclusion}

A high-sensitivity MSF temperature sensor is proposed in this paper. Both the gold and PDMS are deposited outer surface of MSF to minimize the fabrication complexity and achieve more efficient sensing. The FEM method is used to investigate sensing characteristics of the temperature sensor. At optimized sensor parameters, simulation results show that average sensitivity of the proposed sensor is $9.287 \mathrm{~nm} /{ }^{\circ} \mathrm{C}$ as temperature changes from $-20^{\circ} \mathrm{C}$ to $40^{\circ} \mathrm{C}$, and the maximum sensitivity reaching $13 \mathrm{~nm} /{ }^{\circ} \mathrm{C}$. Owing to its high sensitivity and wide sensing range, the proposed sensor supports temperature detection in intelligent monitoring and industrial production.

\section{Funding}

This work is supported by National Natural Science Foundation of China (Grant No.12074331), National Key Research and Development Project (Grant No. 2019YFB2204001).

Disclosures The authors declare no conflicts of interest.

Ethical Approval Approval was obtained from the ethics committee of YanShan University. The procedures used in this study adhere to the tenets of the Declaration of Helsinki.

Consent to participate Informed consent was obtained from all individual participants included in the study. 
Consent for publication The participant has consented to the submission of the case report to the journal.

\section{Availability of data and materials Data are} available on request to the authors.

\section{Code availability Not applicable}

Authors Contributions All authors contributed to the study conception and design. Material preparation, data collection and analysis were performed by Yuhui Feng, Shuguang Li, Hongyu Li, Xiaojian Meng, and Mengqiang Li. The first draft of the manuscript was written by Yuhui Feng and all authors commented on previous versions of the manuscript. All authors read and approved the final manuscript.

Acknowledgements Authors are thankful to the group members of modeling and simulation laboratory for the fruitful discussions.

\section{Compliance with Ethical Standards}

Conflict of Interests The authors declare that they have no conflicts of interest.

\section{References}

[1] J. Guo, Y. Zhong, Y. Liu, et al., A novel high birefringence equal diameter circular-hole photonic crystal fiber, Optoelectronics Letters. 05 (2017) 34-38.

[2] F. Gao, N. Song, X. Xu, et al., Low-loss hollow-core photonic bandgap fiber with isolated anti-resonance layer, Optics Communications. 441 (2019) 208-211.

[3] T. K. Yadav, R. Narayanaswamy, M. H. A. Bakar, et al., Single mode tapered fiber-optic interferometer based refractive index sensor and its application to protein sensing, Optics Express. 22 (19) (2014) 22802-22807.

[4] X. Yu, Y. Zhang, S. Pan, et al., A selectively coated photonic crystal fiber based surface plasmon resonance sensor, Journal of Optics. 12 (1) (2009) 015005.

[5] J. Xue, S. Li, Y. Xiao, et al., Polarization filter characters of the gold-coated and the liquid filled photonic crystal fiber based on surface plasmon resonance, Optics Express. 21 (11) (2013) 13733-13740. [6] W. Yao, Y. Zhu, S. Wang, et al., Flat-topped hollow laser beams can be obtained by using the multi-core photonic crystal fiber mixed with $\mathrm{GeO} 2$, Optik International Journal for Light \& Electron Optics. 126 (23) (2015) 3761-3766.

[7] M. Hautakorpi, M. Mattinen, H. Ludvigsen, Surface-plasmon-resonance sensor based on three-hole microstructured optical fiber, Optics Express. 16 (12) (2008) 8427-8432.

[8] B. Shuai, L. Xia, Y. Zhang, et al., A multi-core holey fiber based plasmonic sensor with large detection range and high linearity, Optics Express. 20 (6) (2012) 5974-5986.

[9] W. Wang, Z. Xiong, W. Shi, et al., Fiber-Optic Surface Plasmon Resonance Sensing Technology, Laser \& Optoelectronics Progress. 54 (9) (2017) 090008.

[10] X. Wang, X. Hong, S. Zhan, et al., Surface Plasmon Resonance Sensing Technology and Bioanalytical Instrument, Progress in Chemistry -Beijing. 26 (7) (2014) 1143-1159.

[11] X. Yang, Y. Lu, B. Liu, J. Yao, Temperature Sensor Based on Photonic Crystal Fiber Filled With Liquid and Silver Nanowires, IEEE Photonics Journal. 8 (3) (2016) 1-1.

[12] Y. Zhao, Z. Deng, H. Hu. Fiber-Optic SPR Sensor for Temperature Measurement, IEEE Transactions on Instrumentation \& Measurement. 64 (11) (2015) 3099-3104.

[13] N. Luan, C. Ding, J. Yao. A Refractive Index and Temperature Sensor Based on Surface Plasmon Resonance in an Exposed-Core Microstructured Optical Fiber, IEEE Photonics Journal. 8 (2) (2016) 1-1.

[14] M. Lu, X. Zhang, Y. Liang, et al., Liquid crystal filled surface plasmon resonance thermometer, Optics Express. 24 (10) (2016) 10904.

[15] C. Zhu, C. Tan, Y. Wang, et al., Photonic Crystal Fiber High Sensitivity Magnetic Field and Temperature Sensor Based on Surface Plasma Resonance Effect and Defect Coupling, Chinese Journal of Lasers. 03 (2017) 253-261.

[16] X. Liu, H. Cheng, Z. Jiao, et al., Temperature sensing characteristics of symmetric structured photonic 
crystals in optical fibers, Laser \& Infrared. 49 (04) (2019) 477-481.

[17] A. Samoc, Dispersion of refractive properties of solvents: Chloroform, toluene, benzene, and carbon disulfide in ultraviolet, visible, and near-infrared, Journal of Applied Physics. 94 (9) (2003) 6167-6174.

[18] A. Vial, A. S. Grimault, D. Macias, et al., Improved analytical fit of gold dispersion:Application to the modeling of extinction spectra with a finite-difference time-domain method, Physical Review B Condensed Matter. 71 (8) (2005) 85416.

[19] Y. Fainman, L. Lee, D. Psaltis, et al., Optofluidics: Fundamentals, Devices, and Applications, McGraw-Hill. (2010).

[20] X. Zhang, Y. Yu, C. Zhu, et al., Miniature End-Capped Fiber Sensor for Refractive Index and Temperature Measurement, IEEE Photonics Technology Letters. 26 (1) (2013) 7-10.

[21] C. S. Park, K. I. Joo, S. W. Kang, et al., A PDMS-Coated Optical Fiber Bragg Grating Sensor for Enhancing Temperature Sensitivity, Journal of the Optical Society of Korea. 15 (4) (2011) 329-334.

[22] Hernández-Romano, et al., Optical fiber temperature sensor based on a microcavity with polymer overlay, Optics Express. 24 (5) (2016) 5654.

[23] J. N. Dash, R. Jha, On the Performance of Graphene-Based D-Shaped Photonic Crystal Fibre Biosensor Using Surface Plasmon Resonance, Plasmonics. 10 (5) (2015) 1123-1131.

[24] X. Wang, S. Li, H. Chen, et al., Compatibility of Temperature Sensor and Polarization Filter Based on $\mathrm{Au}$ Film and Glycerin Selectively Infilling Photonic Crystal Fibers, Plasmonics. 11 (5) (2016) 1-7.

[25] H. Liu, B. Bai, Y. Zhang, et al., High-sensitivity temperature measurement of gold-PDMS-coated photonic crystal fiber based on SPR effect, Chinese Journal of Lasers. 04 (2020) 210-216.

[26] Y. Peng, J. Hou, Z. Huang, et al., Temperature sensor based on surface plasmon resonance within selectively coated photonic crystal fiber, Applied Optics. 51 (26) (2012) 6361-6367.

[27] X. Li, Y. Zhao, X. Zhou, et al., High sensitivity all-fiber Sagnac interferometer temperature sensor using a selective ethanolfilled photonic crystal fiber, Instrumentation Science \& Technology. 46 (3) (2018).

[28] Y. Geng, X. Li, X. Tan, et al., Compact and Ultrasensitive Temperature Sensor With a Fully Liquid-Filled Photonic Crystal Fiber Mach-Zehnder Interferometer, IEEE Sensors Journal. 14 (1) (2013) $167-170$

[29] Z. Liu, L. Liu, Z. Zhu, et al., Whispering gallery mode temperature sensor of liquid microresonastor, Optics Letters. 41 (20) (2016) 4649.

[30] Y. J. Wang, S. G. Li, H. L. Chen, J. S. Li, M. Shi, S. H. Zhang, Z. Zhang. Rectangular Lattice Photonic Crystal Fiber with Simultaneous Polarization Filtering at Communication Wavelengths of $1310 \mathrm{~nm}$ and $1550 \mathrm{~nm}$, Photonics and Nanostructures - Fundamentals and Applications. 33 (2019) 36-41.

[31] Y. J. Wang, S. G. Li, J. J. Wu, P. T. Yu, Z. H. Li. Design of an ultrabroadband and compact filter based on square-lattice photonic crystal fiber with two large gold-coated air holes, Photonics and Nanostructures Fundamentals and Applications. 41 (2020) 100816.

[32] H. Y. Li, S. G. Li, S. Wang, S. Zhang. Theoretical analysis of a microstructured fiber temperature sensor based on a Sagnac interferometer with a wide temperature measurement range, Photonics and Nanostructures Fundamentals and Applications. 43 (2021) 100858. 